Virginia Commonwealth University

vCU Scholars Compass

2012

\title{
Adsorption of oxygen-containing functional groups on free and supported graphene using point contact
}

\author{
Qian Wang \\ Peking University \\ D.X. Ye \\ Virginia Commonwealth University \\ Y. Kawazoe \\ Tohoku University \\ Puru Jena \\ Virginia Commonwealth University, pjena@vcu.edu
}

Follow this and additional works at: http://scholarscompass.vcu.edu/phys_pubs

Part of the Physics Commons

Wang, Q. Ye, D.X., Kawazoe, Y., et al. Adsorption of oxygen-containing functional groups on free and supported graphene using point contact. Physical Review B, 85, 085404 (2012). Copyright (C 2012 American Physical Society.

\section{Downloaded from}

http://scholarscompass.vcu.edu/phys_pubs/57

This Article is brought to you for free and open access by the Dept. of Physics at VCU Scholars Compass. It has been accepted for inclusion in Physics Publications by an authorized administrator of VCU Scholars Compass. For more information, please contact libcompass@vcu.edu. 


\title{
Adsorption of oxygen-containing functional groups on free and supported graphene using point contact
}

\author{
Qian Wang, ${ }^{1,2, *}$ D. X. Ye, ${ }^{2}$ Y. Kawazoe, ${ }^{3}$ and P. Jena ${ }^{2}$ \\ ${ }^{1}$ Center for Applied Physics and Technology, College of Engineering, Peking University, Beijing 100871, China \\ ${ }^{2}$ Physics Department, Virginia Commonwealth University, Richmond, VA 23284, USA \\ ${ }^{3}$ Institute for Material Research, Tohoku University, Sendai, 980-8577, Japan
}

(Received 28 July 2011; revised manuscript received 1 December 2011; published 3 February 2012)

\begin{abstract}
First-principles electronic structure calculations based on spin-polarized density functional theory were carried out to study the adsorption of oxygen-containing functional groups - $\mathrm{OH},-\mathrm{CHO}$, and - $\mathrm{COOH}$ on a two-dimensional (2D) infinite graphene sheet without edge states and defects. We find that the energy gain of adsorption can be significantly improved when the graphene sheet is supported via a point contact, a prototype for graphene sheet supported by catalysts, nanoparticles or nanopillars, or a surface with steps, edges, adatoms, or defects. This was modeled by placing a single atom of $\mathrm{Fe}, \mathrm{Co}$, and $\mathrm{Ni}$ under the graphene surface. Thus supported graphene not only becomes magnetic, but the carbon atoms in contact with the metal atoms become chemically more active as well. The use of point contact support to improve adsorption has advantages over that of introducing defects: (1) It does not destroy the intrinsic 2D geometry of the graphene sheet. (2) Patterned structures can be created by tailoring the position of the metal atoms supporting the graphene sheet. (3) The geometry distortion created by point contact can be made more uniform and lead to better adsorption energies. (4) Reduction of the work function of supported graphene makes the manipulation of electrons more flexible and controllable in tuning their electronic structure and magnetic properties.
\end{abstract}

DOI: 10.1103/PhysRevB.85.085404

PACS number(s): 81.05.ue, 73.22.Pr, 31.15.A-

\section{INTRODUCTION}

Graphene is one-atom-thick $s p^{2}$-bonded carbon atoms arranged in a honeycomb hexagonal planar sheet. As a novel nanomaterial and as the basic building block for graphitic materials of all other dimensionalities [zero-dimensional (0D) fullerenes, one-dimensional (1D) nanotubes, and 3D graphite], it has currently attracted tremendous attention from chemists, physicists, engineers, and materials scientists aiming to provide a fundamental insight into all carbon materials and to explore various promising technological applications based on its exceptional properties, such as excellent electrical conductivity, high surface-to-volume ratio, remarkable mechanical strength, and biocompatibility. ${ }^{1-3}$ Among various research directions, functionalization of graphene is extensively pursued for the following reasons: (1) Engineering the band gap and modulating the electronic structure: Graphene is metallic-like with zero band gap, which needs to be tuned for microelectronic applications. ${ }^{4,5}$ (2) Modifying chemical properties: Functionalization of graphene is expected to promote chemical reactivity. For example, the hydrophobic graphene can become hydrophilic when functionalized. ${ }^{6}$ (3) Improving the role of graphene as sensors: By suitably choosing the support, interaction of graphene toward molecules and biomolecules could be enhanced. ${ }^{7}$ (4) Assembling graphene with superstructures and preventing aggregations of graphene sheets (GSs). ${ }^{8}$ Among various functional complexes, oxygen-containing functional groups (FGs) are of special interest because their unpaired electrons can make them highly reactive, and such functionalized graphene-based materials provide unprecedented opportunities for further functionalization because the derivative FGs are able to bind with other designated molecules.

It was found that the functionalization of graphene and carbon nanoribbons preferentially occurs at defect sites introduced by acid treatment ${ }^{9-13}$ or at the edge sites of nanoribbons, which substantially change the structural and electronic characteristics of defective graphene-based materials. ${ }^{14-16} \mathrm{Al}$ though defects can enhance binding with FGs, they also change the intrinsic properties of graphene. With the advancement of experimental techniques, it has been one of the current research directions to synthesize defect-free GSs. We note that for a perfect 2D GS without any defects and edge states, the functionalization would be different. Thus, questions arise: For a defect-free GS, which FGs can effectively bind with GSs? How does functionalization affect the electronic structure and magnetic properties of GSs? How can one tune the interaction between GSs and FGs to form patterned adsorptions?

In this paper, we focus on the study of functionalization of GSs using oxygen-containing radicals, such as the hydroxyl radical $(-\mathrm{OH})$, aldehyde group $(-\mathrm{CHO})$ and carboxyl group $(-\mathrm{COOH})$, to manipulate the physical and chemical properties of graphene because these radicals play important roles in living systems, biochemistry, and medicine. We have found that the $-\mathrm{OH}$ group can bind strongly with pristine graphene, whereas the binding with $-\mathrm{CHO}$ and $-\mathrm{COOH}$ is weak. This can be enhanced when the GS is supported via a point contact. We model this by using transition metal (TM = Fe, Co, and $\mathrm{Ni}$ ) atoms as support. We note that the TM atoms are electron donors, even though their electron ionization potentials are much larger than the work function of GSs. Thus, the point contact changes the electronic structure and properties of graphene and enhances the adsorption of the FGs.

\section{THEORETICAL METHOD}

Our study is based on spin-polarized density functional theory (DFT) with generalized gradient approximation (GGA) for exchange and correlation. We used the supercell approach, wherein a graphene sheet was modeled by $3 \times 3$ graphene unit 
cells (containing 18 atoms) with a vacuum space of $18 \AA$ in the normal direction (i.e. the $z$ direction of the graphene plane), which is large enough to ensure no interaction between adjacent layers. Calculations of total energies, forces, and optimization of geometries were carried out using a plane wave basis set and the projector augmented wave method ${ }^{17}$ as implemented in the Vienna ab initio Simulation Package. ${ }^{18}$ The PW91 functional ${ }^{19}$ was used for the exchange and correlation potential. The Brillouin zone was represented by a Monkhorst-Pack (MP) special k-point mesh ${ }^{20}$ of $19 \times 19 \times 1$, and the Gaussian smearing with a width of $0.02 \mathrm{eV}$ was used for the occupied electronic levels. Tests were also performed by increasing MP grids up to $25 \times 25 \times 1$ to ensure the convergence of all properties of the system. The resulting difference in total energies was found to be less than $5 \mathrm{meV} /$ supercell in both the pristine and TM-supported graphene sheets.

To study the effect of distribution and concentration of FGs on the electronic and magnetic properties, calculations were also carried out for a $4 \times 4$ supercell containing 32 carbon atoms. In the calculations, the atomic coordinates of all the atoms were relaxed without any symmetry constraint. We used $15 \times 15 \times 1$ and $19 \times 19 \times 1$ MP grids, respectively, for the structure optimization and for total energy and spin density calculations. The cutoff energy was set to $450 \mathrm{eV}$ for the planewave basis (the default of maximum cutoff energy is $400 \mathrm{eV}$ ). Self-consistency was achieved with a tolerance in the total energy of $3 \times 10^{-4} \mathrm{eV}$. Hellman-Feynman force components on each ion in the supercell were converged to $3 \times 10^{-3} \mathrm{eV} / \AA$. The accuracy of our computational procedure is tested using the pristine GS supercells. The optimized bond length of C-C of $1.43 \AA$ is in good agreement with the experimental value of $1.42 \AA$. The band structure is plotted in Fig. 1, which is also in good agreement with previously calculated results. ${ }^{5}$

To study the adsorption of FGs on GSs qualitatively, we define the adsorption energy $E_{\mathrm{a}}$ as the energy difference between the complex and the individual units, i.e.,

$$
\begin{gathered}
\left.E_{\mathrm{a} 1}=-\left[E_{\mathrm{GS}-\mathrm{FG}}-E_{\mathrm{GS}}-E_{\mathrm{FG}}\right] \quad \text { (the adsorption energy of a } \mathrm{FG} \text { on a free-standing } \mathrm{GS}\right), \\
\left.E_{\mathrm{b}}=-\left[E_{\mathrm{TM}-\mathrm{GS}}-E_{\mathrm{GS}}-E_{\mathrm{TM}}\right] \quad \text { (the binding energy of a TM atom on a free-standing } \mathrm{GS}\right), \\
E_{\mathrm{a} 2}=-\left[E_{\mathrm{TM}-\mathrm{GS}-\mathrm{FG}}-E_{\mathrm{TM}-\mathrm{GS}}-E_{\mathrm{FG}}\right] \quad \text { (the adsorption energy of a FG on the TM-supported GS), }
\end{gathered}
$$

where $E_{\mathrm{GS}-\mathrm{FG}}, E_{\mathrm{TM}-\mathrm{GS}}$, and $E_{\mathrm{TM}-\mathrm{GS}-\mathrm{FG}}$ are the total energies of the GS supercell with a FG, the GS supercell with a TM atom, and the TM-supported GS with a FG, respectively. $E_{\mathrm{TM}}$, $E_{\mathrm{GS}}$, and $E_{\mathrm{FG}}$ are the total energies of an isolated TM atom, the pristine GS supercell, and an isolated FG. The total energy of an isolated atom or molecule is calculated with the single

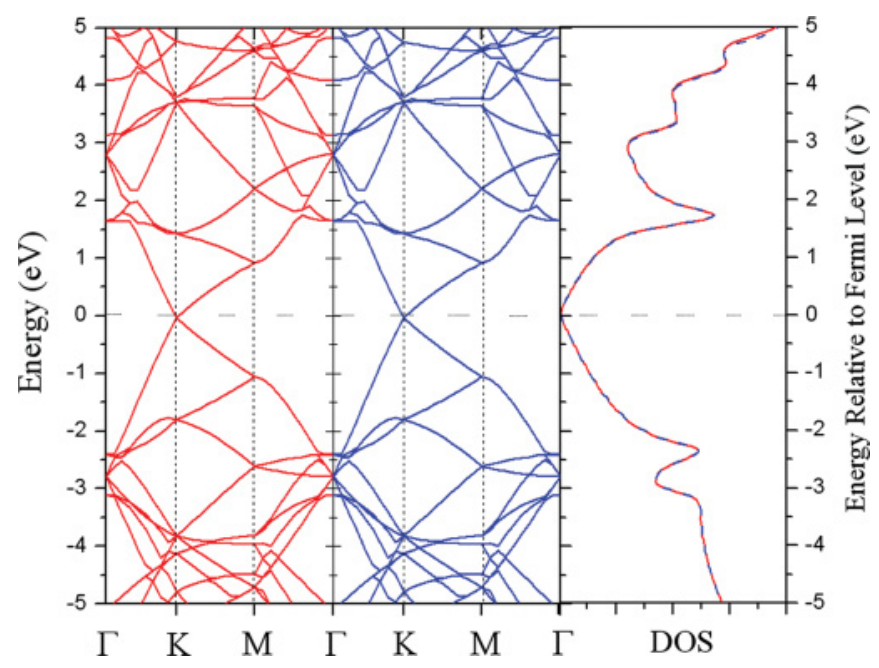

FIG. 1. (Color online) Calculated band structures and the corresponding total DOS plot for the majority (red lines) and minority (blue lines) spin configurations for the $4 \times 4$ graphene supercell. $\Gamma$ $(0,0,0), K(-1 / 3,2 / 3,0)$, and $M(0,1 / 2,0)$ are highly symmetric points of the reciprocal space, and $K$ is the Dirac point of the graphene sheet. The DOS is calculated using a $15 \times 15 \times 1 \mathrm{MP}$ grid to sample the Brillouin zone, and the graph is smoothed by Gaussian smearing of width $0.15 \mathrm{eV}$. atom or molecule in a cubic supercell of length $15 \AA$. The total energies were computed with the spin-polarized calculations.

\section{RESULTS AND DISCUSSIONS}

\section{A. Adsorption of functional groups on pristine graphene}

We first studied the adsorption of FGs on a pristine free-standing GS. We started with the hydroxyl radical $(-\mathrm{OH})$ since it has many important chemical and biomedical applications with flexibility in binding. For example, in alcohols, the hydroxyl group is attached to a carbon atom in a hydrocarbon chain, whereas in phenols, the hydroxyl group is attached directly to the hydrocarbon ring. Although recently the adsorption of $-\mathrm{OH}$ and other FGs on pristine and defected graphene has been theoretically studied, ${ }^{21-28}$ the details of the interaction have not yet been fully understood, and the calculated adsorption energy and magnetic moment for $-\mathrm{OH}$ individually adsorbed on pristine graphene are quite different, ranging from 0.21 to $0.70 \mathrm{eV}$ and 0.00 to $0.75 \mu_{\mathrm{B}}$, respectively. Here, we started by considering the three high-symmetry adsorption sites on the GS: a hollow $(\mathrm{H})$ site above the center of a carbon hexagon ring, a bridge (B) site above the carbon-carbon bond, and an on-top (T) site above the $\mathrm{C}$ atom with different initial orientations of $-\mathrm{OH}$ radical, respectively. Each initial structure was optimized without any symmetry constraint. The lowest energy configuration of the hydroxylated graphene is represented in Fig. 2(a1). We found that the hydroxyl radical prefers to bind to GSs at the on-top $\mathrm{C}$ site with the O-C bond length $d_{\mathrm{O}-\mathrm{C}}$ of $1.511 \AA$ and the adsorption energy of $0.712 \mathrm{eV}$, which is comparable to those of 0.70 and $0.67 \mathrm{eV}$ obtained by Lahaye et al. ${ }^{21}$ and Kim et al. ${ }^{22}$ respectively. The -OH group moves away (about $3.5 \AA$ ) from 

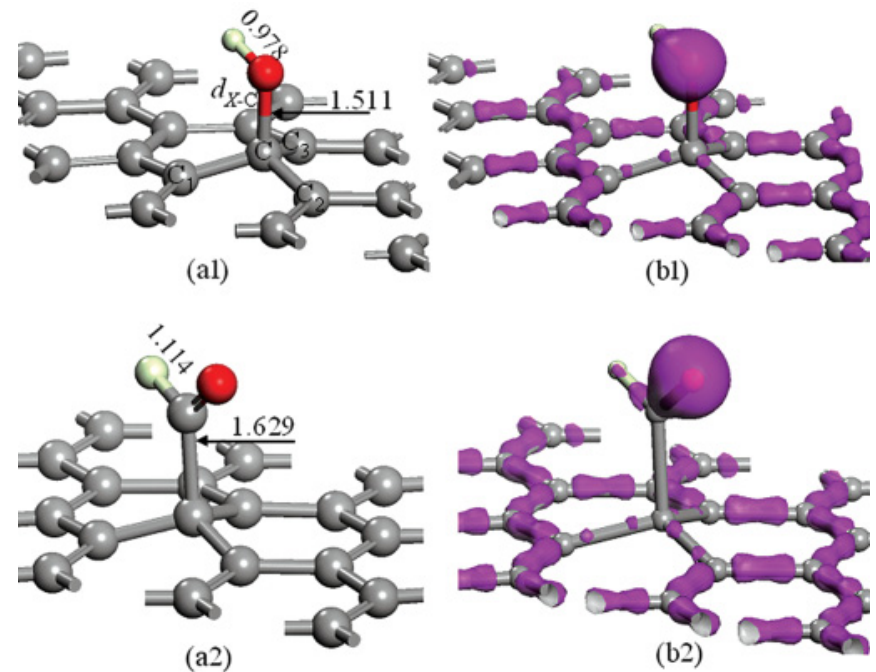

(a2)

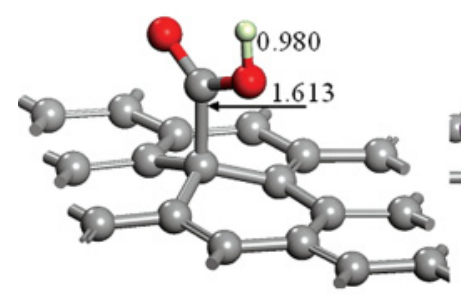

(a3)

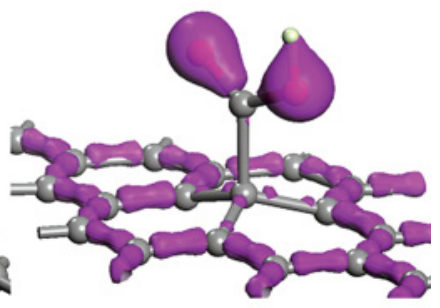

(b3)

FIG. 2. (Color online) The lowest energy configurations (a1)-(a3) and their corresponding 3D charge density isosurfaces (b1)-(b3) with a value of 1.90 electrons $/ \AA^{3}$ for $-\mathrm{OH}$, $-\mathrm{CHO}$, and - $\mathrm{COOH}$ adsorbed on pristine GS. The unit of the bond lengths in (a1)-(a3) is $\AA$.

the graphene plane when it is introduced on the $\mathrm{H}$ site or the $\mathrm{B}$ site. The adsorption leads to a significant change of the local geometry, and a quasi-tetrahedral bonding configuration of the $\mathrm{C}$ atoms is formed at the adsorption site having $s p^{3}$ hybrid bonding. The $\mathrm{C}$ atom at an adsorption site is pulled out of the plane, and the $\mathrm{C}-\mathrm{C}$ bond length is elongated. The average bond length between the $C$ and its neighbors $\left(C_{1}, C_{2}\right.$, and $\mathrm{C}_{3}$ ), as shown in Fig. 2(a1), increases from 1.420 to $1.494 \AA$. We quantify the distortion of GSs $\left(d_{\mathrm{GZ}}\right)$ by calculating the maximum deviation in the $z$ direction of the adsorbing-site $\mathrm{C}$ atom from its position in the pristine GS, which is $0.427 \AA$. Furthermore, the adsorption not only affects the geometry of the substrate, but also its electronic structure, which can be seen from the charge density isosurface plotted in Fig. 2(b1), showing the charge distribution of the - $\mathrm{OH}$ adsorbed on GSs. We note that the covalent bonding feature of $\mathrm{C}-\mathrm{C}$ became weaker for the bonds near the - $\mathrm{OH}$ group because of the charge redistribution caused by the adsorption of FGs.

We then studied the adsorption of another two, different oxygen-containing FGs, namely, the aldehyde group (-CHO) and carboxyl group (-COOH), on the pristine GS. Although these two molecules also contain oxygen, they are different from the $-\mathrm{OH}$ group. According to conventional chemistry, when using a benzene molecule as the reference, $-\mathrm{OH}$ has an activating ability, and it is a strong electron-donating group, whereas - $\mathrm{CHO}$ and $-\mathrm{COOH}$ are moderate electronwithdrawing groups, showing deactivating behavior. It would be interesting to investigate when a benzene molecule is extended to a 2D GS to see whether such differences still exist. Our calculations suggest that both $-\mathrm{CHO}$ and $-\mathrm{COOH}$ still prefer the on-top $\mathrm{C}$ site configuration as in the $-\mathrm{OH}$ case [see Fig. 2(a2) and 2(a3)]. However, the corresponding adsorption energies are found to be only 0.033 and $0.025 \mathrm{eV}$, respectively. Therefore, the adsorption seems very weak. On the other hand, the adsorption distance between the $\mathrm{C}$ atom in the FG and the adsorption site $\mathrm{C}$ on the GS is found to be 1.629 and $1.613 \AA$, respectively, which are still within the $\mathrm{C}-\mathrm{C}$ bonding distance. The adsorption results in some visible local distortion of the GS. In diamond, the $\mathrm{C}-\mathrm{C}$ bond is $1.54 \AA$, whereas in cyclobutabenzene, $\mathrm{C}-\mathrm{C}$ bond length can reach $1.74 \AA$ based on x-ray crystallography. ${ }^{29}$ A long C-C bond length of up to $2.90 \AA$ has also been observed in a dimer of two tetracyanoethylenedian, ${ }^{30}$ and even a bond length of $3.05 \AA$ exists in pancake bond structure. ${ }^{31}$ On the basis of these findings, we can see that carbon atoms exhibit very flexible bonding behavior in different bonding environments. Even though the adsorption of - $\mathrm{CHO}$ and $-\mathrm{COOH}$ groups produces significant distortion of the GS, the small binding energy occurs because the energy cost from the geometry distortion compensates the energy gain upon adsorption.

It is well known that charge transfer sensitively depends on the bonding environment. In this work, we determined charge transfer in two ways. One is based on the shift in Fermi energy ( $E_{\mathrm{F}}$, using vacuum level as the zero-energy reference) in the density of states (DOS) resulting from the adsorption of FGs on the GS. The other is the Wigner-Seitz cell method, which can be used for charge partitioning. The charge transfers are derived by calculating the charge difference between a given complex and the individual units. In this way, the errors from partitioning will cancel to some extent. We calculated $E_{\mathrm{F}}$ for the pristine GS and the FG-adsorbed GS to determine whether electrons from these FGs are transferred to the graphene substrate. It was found that $E_{\mathrm{F}}$ varies from $-2.105 \mathrm{eV}$ for the pristine GS to $-2.502,-2.054$, and $-1.853 \mathrm{eV}$ for the -OH-, -CHO-, and -COOH-adsorbed GSs, respectively, which indicates that the hydroxyl radicals act as electron acceptors, withdrawing electrons from the GS, whereas the aldehyde group and carboxyl group behave as electron donors. Charge analysis further suggests that the $-\mathrm{OH}$ withdraws $-0.167 e$ $(-0.430 e$ at the $\mathrm{O}$ site and $0.263 e$ at the $\mathrm{H}$ site) from the $\mathrm{GS}$, whereas the $-\mathrm{CHO}$ and $-\mathrm{COOH}$ donate 0.125 and $0.090 e$, respectively, to the $\mathrm{GS}$ (in $-\mathrm{CHO}, 0.328 e$ at the $\mathrm{C}$ site, $0.081 e$ at the $\mathrm{H}$ site, and $-0.284 e$ at the $\mathrm{O}$ site; in $-\mathrm{COOH}$, $0.568 e$ at the $\mathrm{C}$ site, $0.438 e$ at the $\mathrm{H}$ site, and $-0.372 e$ and $-0.544 e$ at the two $\mathrm{O}$ sites, respectively). Therefore, these three FGs exhibit different bonding behavior when adsorbed on the 2D GS from that on the benzene molecules. In Fig. 2(b1), 2(b2), and 2(b3), we show the electron density distributions. We note that the $\mathrm{C}-\mathrm{C}$ covalent bonding in the GS is weakened because of the charge transfer between the GS and the FGs.

To further study the effect of concentration and distribution of the FGs on the adsorption and electronic structure, we carried out additional calculations by changing the supercell size to $4 \times 4$, which contains 32 carbon atoms and ensures a large distance of about $10 \AA$ for the separation between neighboring FGs in the $x y$ plane. Following the same procedure as described above, once again, we found that the FGs prefer to 
TABLE I. The adsorption energy $\left(E_{\mathrm{a} 1}\right)$, adsorption distance $\left[d_{\mathrm{X}-\mathrm{C}}\right.$, the distance between the $\mathrm{C}$ atom at the adsorption site and its bonded atom $(\mathrm{X})$ in the FG], the average bond length $\left(d_{\mathrm{C}-\mathrm{C}}\right)$ between the $\mathrm{C}$ atom at the adsorption site and its nearest neighbor [i.e., $\mathrm{C}_{1}, \mathrm{C}_{2}$, and $\mathrm{C}_{3}$, as shown in Fig. 2(a)], the distortion of GSs $\left(d_{\mathrm{GZ}}\right)$ measured by calculating the maximum deviation in the $\mathrm{z}$ direction of the $\mathrm{C}$ atom from its original position in the pristine GS, Fermi energy $\left(E_{\mathrm{F}}\right)$, and charge transfer $(\Delta Q)$ from the FGs to GSs for each of the ground state configurations of the $\mathrm{C}_{18}-\mathrm{FG}(\mathrm{FG}=-\mathrm{OH},-\mathrm{CHO}$, and $-\mathrm{COOH})$. The data for the $(4 \times 4)$ supercell, $\mathrm{C}_{32}-\mathrm{FG}$, is given in parentheses for comparison.

\begin{tabular}{lccccccc}
\hline \hline System & $\begin{array}{c}E_{\mathrm{a} 1} \\
(\mathrm{eV})\end{array}$ & $\begin{array}{c}d_{\mathrm{X}-\mathrm{C}} \\
(\AA)\end{array}$ & $\begin{array}{c}d_{\mathrm{C}-\mathrm{C}} \\
(\AA)\end{array}$ & $\begin{array}{c}d_{\mathrm{GZ}} \\
(\AA)\end{array}$ & $\begin{array}{c}E_{\mathrm{F}} \\
(\mathrm{eV})\end{array}$ & $\begin{array}{c}\Delta Q \\
(e)\end{array}$ \\
\hline $\mathrm{C}_{18}-\mathrm{OH}$ & 0.712 & 1.511 & 1.494 & 0.427 & -2.502 & -0.167 \\
$\left(\mathrm{C}_{32}-\mathrm{OH}\right)$ & $(0.798)$ & $(1.510)$ & $(1.496)$ & $(0.468)$ & $(-2.472)$ & $(-0.179)$ \\
$\mathrm{C}_{18}-\mathrm{CHO}$ & 0.033 & 1.629 & 1.489 & 0.356 & -2.054 & 0.125 \\
$\left(\mathrm{C}_{32}-\mathrm{CHO}\right)$ & $(0.039)$ & $(1.626)$ & $(1.491)$ & $(0.386)$ & $(-2.084)$ & $(0.114)$ \\
$\mathrm{C}_{18}-\mathrm{COOH}$ & 0.025 & 1.613 & 1.503 & 0.392 & -1.853 & 0.090 \\
$\left(\mathrm{C}_{32}-\mathrm{COOH}\right)$ & $(0.026)$ & $(1.608)$ & $(1.504)$ & $(0.415)$ & $(-1.949)$ & $(0.088)$ \\
\hline \hline
\end{tabular}

reside at the on-top C sites. Very similar results are obtained using this larger supercell; namely, the adsorption of $-\mathrm{OH}$ on the GS is much stronger than that of $-\mathrm{CHO}$ and $-\mathrm{COOH}$ on the GS. The $E_{\mathrm{F}}$ of the hydroxyl-functionalized GS is found to be $-2.472 \mathrm{eV}$, which is less than that of $-2.104 \mathrm{eV}$ for the pristine GS, withdrawing 0.179 electrons from the GS, according to charge population analysis. The $E_{\mathrm{F}}$ values for the aldehyde GS and carboxylated GS are, respectively, -2.084 and $-1.949 \mathrm{eV}$. Because these values are greater than that of the pristine GS, they donate 0.114 and 0.088 electrons, respectively, to the GS. The adsorption distance $d_{\mathrm{X}-\mathrm{C}}$ lies in the range of 1.51 $1.63 \AA$, whereas the amount of GS distortion $d_{\mathrm{GZ}}$ lies in the range of $0.39 \sim 0.47 \AA$. The hydroxyl radical has a shorter adsorption distance and causes a larger distortion of the GS because of the stronger binding with GSs compared with the aldehyde and carboxyl groups. The main results for the ground state configurations corresponding to each of the FG-adsorbed GSs are summarized in Table I.

We note that adsorption of the FGs on GSs induces a nonvanishing magnetic moment. This is because all three FGs

TABLE II. The binding energy $\left(E_{\mathrm{b}}\right)$, total magnetic moment $(M)$, average bond length between the TM atom and its nearing $\mathrm{C}$ atoms in the hexagon $\left(d_{\mathrm{TM}-\mathrm{C}}\right)$, TM-to-graphene plane distance $\left(d_{\mathrm{TM}-\mathrm{G}}\right)$, graphene distortion $\left(d_{\mathrm{GZ}}\right)$, Fermi energy $\left(E_{\mathrm{F}}\right)$, and charge transferred $(\Delta Q)$ from TM to GSs corresponding to the lowest energy configuration in the $(3 \times 3)$ supercell, $\mathrm{TM}-\mathrm{C}_{18}(\mathrm{TM}=\mathrm{Fe}, \mathrm{Co}$, and $\mathrm{Ni})$. The numbers in parenthesis are for the $(4 \times 4)$ supercell, TM-C 32 .

\begin{tabular}{lcccccccc}
\hline \hline System & $\begin{array}{c}E_{\mathrm{b}} \\
(\mathrm{eV})\end{array}$ & $\begin{array}{c}M \\
\left(\mu_{\mathrm{B}}\right)\end{array}$ & $\begin{array}{c}d_{\mathrm{TM}-\mathrm{C}} \\
(\AA)\end{array}$ & $\begin{array}{c}d_{\mathrm{TM}-\mathrm{G}} \\
(\AA)\end{array}$ & $\begin{array}{c}d_{\mathrm{GZ}} \\
(\AA)\end{array}$ & $\begin{array}{c}E_{\mathrm{F}} \\
(\mathrm{eV})\end{array}$ & $\begin{array}{c}\Delta Q \\
(e)\end{array}$ \\
\hline $\mathrm{Fe}-\mathrm{C}_{18}$ & 0.916 & 2.00 & 2.106 & 1.538 & 0.019 & -1.112 & 0.227 \\
$\left(\mathrm{Fe}-\mathrm{C}_{32}\right)$ & $(0.859)$ & $(2.00)$ & $(2.109)$ & $(1.538)$ & $(0.018)$ & $(-1.478)$ & $(0.241)$ \\
$\mathrm{Co}-\mathrm{C}_{18}$ & 1.240 & 1.00 & 2.100 & 1.517 & 0.028 & -1.105 & 0.080 \\
$\left(\mathrm{Co}-\mathrm{C}_{32}\right)$ & $(1.190)$ & $(1.00)$ & $(2.100)$ & $(1.513)$ & $(0.029)$ & $(-1.475)$ & $(0.104)$ \\
$\mathrm{Ni}-\mathrm{C}_{18}$ & 1.490 & 0.00 & 2.125 & 1.557 & 0.014 & -1.251 & 0.205 \\
$\left(\mathrm{Ni}_{12}\right)$ & $(1.486)$ & $(0.00)$ & $(2.123)$ & $(1.554)$ & $(0.014)$ & $(-1.511)$ & $(0.220)$ \\
\hline \hline
\end{tabular}

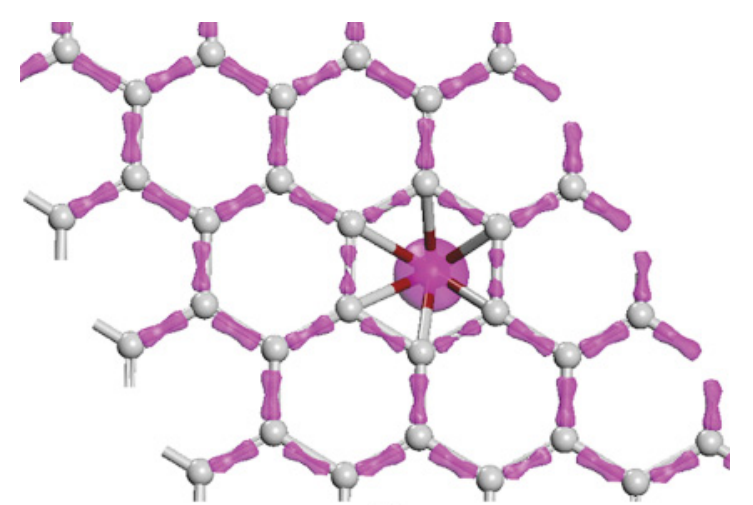

(a)

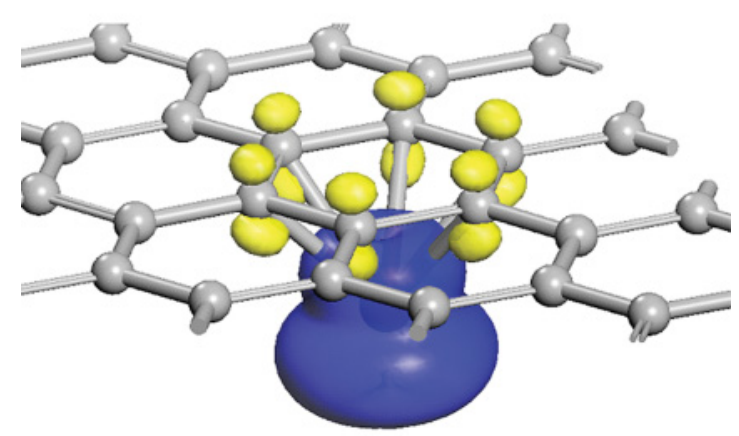

(b)

FIG. 3. (Color online) (a) The charge density isosurface with a value of 1.90 electrons $/ \AA^{3}$ and (b) the corresponding 3D spin density isosurface with a value of 0.03 electrons $/ \AA^{3}$ for the GS supported by Fe point contact.

(-OH, $-\mathrm{CHO}$, and $-\mathrm{COOH}$ ) have one unpaired electron. Thus, the number of electrons in the supercells is odd. In fact, it was found that there is a moment of $1.00 \mu_{\mathrm{B}}$ for each of the GS-CHO and GS-COOH supercells, irrespective of supercell size. The magnetic moment for GS-OH, however, is found to be size dependent (i.e. $0.39 \mu_{\mathrm{B}}$ for $\mathrm{C}_{18}-\mathrm{OH}$ and $0.00 \mu_{\mathrm{B}}$ for $\mathrm{C}_{32}-\mathrm{OH}$ ). We compare this with the previously calculated results, ${ }^{23}$ in which the magnetic moments of $0.87(\mathrm{n}=2), 0.075$ $(\mathrm{n}=3)$, and $0.00 \mu_{\mathrm{B}}(\mathrm{n}=4$ and 6$)$ were found for the $(\mathrm{n} \times \mathrm{n})$ $\mathrm{OH}$ supercells. We carried additional calculations by using the $(2 \times 2)$ and $(6 \times 6)$ supercells containing 8 and 72 carbon atoms, respectively. The convergence test for the MP grids was performed by using the MP k-point meshes of $(19 \times 19 \times 1)$, $(21 \times 21 \times 1),(23 \times 23 \times 1),(25 \times 25 \times 1)$, and $(27 \times 27 \times 1)$ for the $(2 \times 2)$ supercell and $(9 \times 9 \times 1),(11 \times 11 \times 1)$, and $(13 \times 13 \times 1)$ for the $(6 \times 6)$ supercell. We found that the $(25 \times 25 \times 1)$ and $(11 \times 11 \times 1)$ MP $k$-point mesh gave the converged results for the $(2 \times 2)$ and $(6 \times 6)$ supercells, respectively. The magnetic moments are found to be 0.875 and $0.00 \mu_{\mathrm{B}}$ for $\mathrm{C}_{8}-\mathrm{OH}$ and $\mathrm{C}_{72}-\mathrm{OH}$, respectively. The magnetic moments decrease with increasing supercell size, in agreement with previous work. ${ }^{23}$ The smaller magnetic moment calculated for the $(3 \times 3)-\mathrm{OH},{ }^{23}$ namely $\mathrm{C}_{18}-\mathrm{OH}$, may be due to the insufficient MP $k$-point mesh they used because the same result was obtained before the MP grid reached the convergence limit at $(19 \times 19 \times 1)$ in our calculation. When two of the FGs were introduced to the supercells, the total moment was found to be zero. Therefore, 

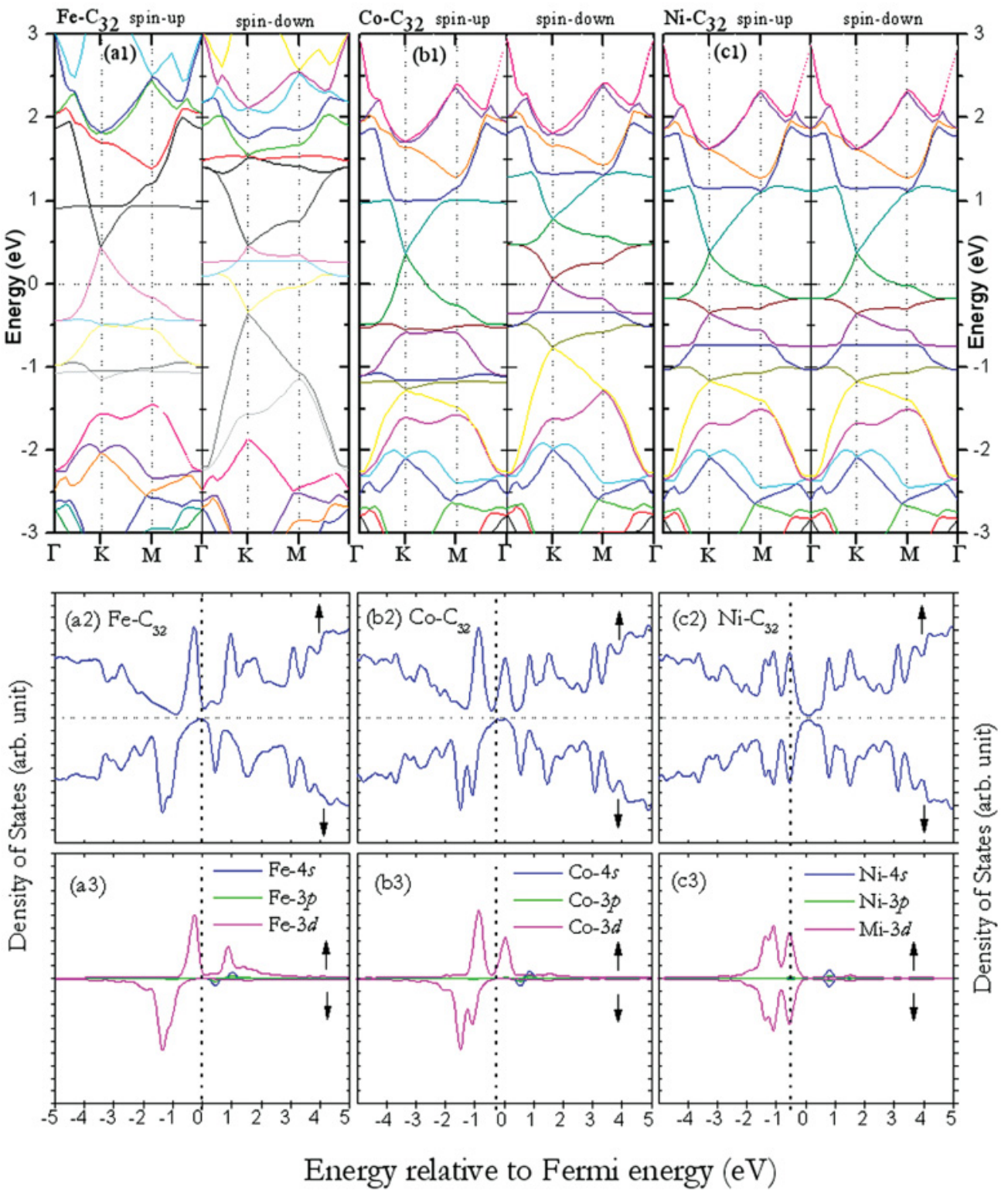

FIG. 4. (Color online) (a1), (b1), (c1) Calculated band structures, (a2), (b2), (c2) the corresponding spin-polarized total DOS, and (a3), (b3), (c3) partial DOS for the $\mathrm{Fe}-\mathrm{C}_{32}, \mathrm{Co}-\mathrm{C}_{32}$, and $\mathrm{Ni}-\mathrm{C}_{32}$ supercells, respectively.

it is impossible to induce a long-range magnetic order in the FG-decorated graphene systems without further modification.

\section{B. Graphene supported by TM point contact}

From the above study, we note that the energy gain of - $\mathrm{CHO}$ or - $\mathrm{COOH}$ on free-standing GSs is weak because of the energy cost associated with the geometric distortion. Therefore, the question arises: How can the energetics be improved?

We recall that in practical applications, graphene cannot be in free-standing mode and supports are necessary. Among the supporting materials, transition metals are widely used as substrates and contacts for GSs. Furthermore, it has been predicated that the Fe/graphene system is stable at $550-600 \mathrm{~K}$, and the system intercalated with Co atoms is stable at temperatures up to $740 \mathrm{~K} .^{32}$ Recently, researchers have considered metal nanoparticles or nanopillars as supports ${ }^{33}$ for GSs. The advantage of this kind of support over the direct deposition of graphene on a metal surface is that the supported system still maintains its nano-size form, and the nano-sized support via point contact leaves space for further functionalization. In fact, point contact is very common and practical. When a GS is deposited on a substrate, the strain caused by the lattice mismatch would result in the rippling of graphene. Furthermore, in most cases, the metal surface contains steps, edges, adatoms, or defects that would further cause distortion of the GS. This can be minimized if the support provides only a point contact that can also improve the reactivity of the $\mathrm{C}$ atoms directly on top of the supporting atom. Current studies have not yet considered such cases. In this study, we have addressed this problem. To reduce computational cost, we use a single atom as the support to model the point contact situations. For point contact, we focus on $\mathrm{Fe}, \mathrm{Co}$, and $\mathrm{Ni}$ atoms because these are often used in experiments. 


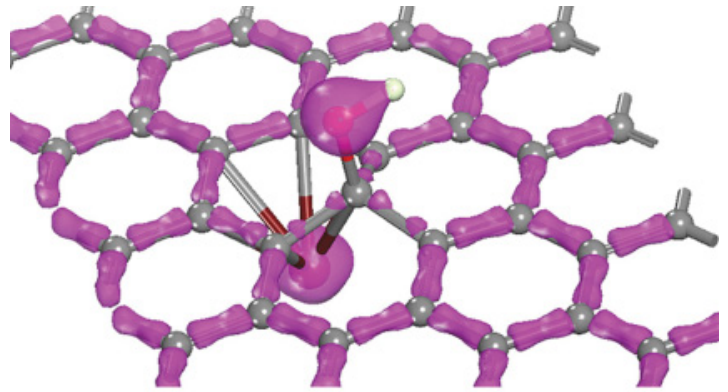

(a)

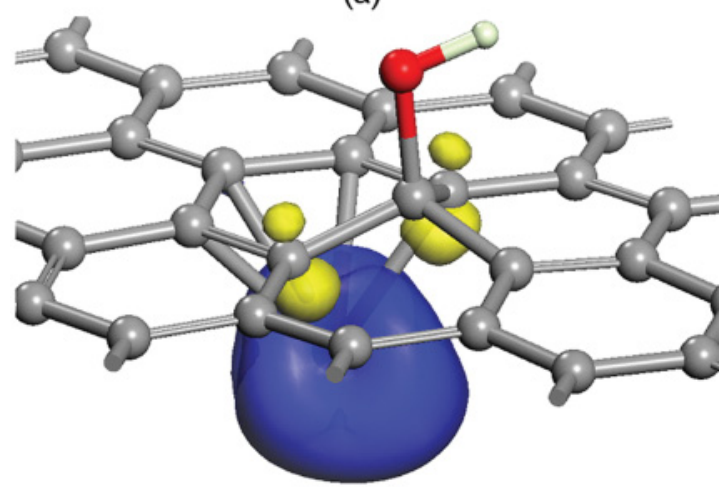

(b)

FIG. 5. (Color online) (a) The charge density isosurface with a value of 1.90 electrons $/ \AA^{3}$ and (b) the corresponding $3 \mathrm{D}$ spin density isosurface with a value of 0.03 electrons $/ \AA^{3}$ for an $-\mathrm{OH}$ on the GS supported by Fe point contact.

Recently, several groups have theoretically studied the adsorption energy and electronic and magnetic properties of TM adatoms adsorbed on graphene. ${ }^{34-43}$ Nevertheless, the results remain controversial. Johll et al. ${ }^{34}$ found the adsorption energies of $0.58,0.97$, and $1.37 \mathrm{eV}$ for a single $\mathrm{Fe}, \mathrm{Co}$, and $\mathrm{Ni}$ adatom on graphene, respectively. These adsorption energies are different from those computed by other authors, namely $0.99,1.58$, and $1.53 \mathrm{eV} ;{ }^{35} 1.21,1.59$, and $1.55 \mathrm{eV} ;^{36}$ and 1.06 , 2.08 , and $2.79 \mathrm{eV}^{37}$ Similarly, the total moment of $3.02 \mu_{\mathrm{B}}$, obtained from DFT at the GGA-PW91 level of theory for Fe-graphene ${ }^{38}$ also differs from the $2 \mu_{\mathrm{B}}$ value obtained by others. ${ }^{34-37,39,41}$ Therefore, we begin with the bonding features of the TM atoms on graphene with the three high-symmetry adsorption sites (i.e. the $\mathrm{H}, \mathrm{B}$, and $\mathrm{T}$ sites). The favored adsorption site is found to be the $\mathrm{H}$ site for all the TM atoms considered here $(\mathrm{TM}=\mathrm{Fe}, \mathrm{Co}$, and $\mathrm{Ni})$. The distance between the TM atoms and the graphene plane $\left(d_{\mathrm{TM}-\mathrm{G}}\right)$ is measured by calculating the difference between the $z$ coordinate of the single TM atom and the average $z$ coordinate of the $\mathrm{C}$ atoms in the graphene supercell. The $d_{\mathrm{TM}-\mathrm{G}}$ is found to be 1.538 , 1.517, and $1.557 \AA$ for the Fe-, Co-, and Ni-adsorbed GSs, respectively, which are in good agreement with the previously calculated values ${ }^{35-37,39,41}$ and also agree with the results of $1.52,1.52$, and $1.53 \AA$ for single $\mathrm{Fe}, \mathrm{Co}$, and $\mathrm{Ni}$ adatoms on a graphite surface. ${ }^{44}$ The distortion $\left(d_{\mathrm{GC}}\right)$ in graphene is determined in the same way as that in the adsorption of FGs on GSs. It is much smaller compared with the results of the adsorption of FGs. The calculated results are listed in Table II.

In the lowest energy configurations of TM- $\mathrm{C}_{18}$, the adsorption distance between the TM atom and its nearest $\mathrm{C}\left(d_{T M-\mathrm{C}}\right)$ was found to be $2.106,2.100$, and $2.125 \AA$ for the Fe-, Co-, and $\mathrm{Ni}$-supported GS, respectively, and the binding energy is found to be $0.916,1.240$, and $1.490 \mathrm{eV}$ for $\mathrm{Fe}, \mathrm{Co}$, and $\mathrm{Ni}$, respectively. The adsorption of $\mathrm{Fe}$ or Co on GSs leads to a net magnetic moment of $2 \mu_{\mathrm{B}}$, or $1 \mu_{\mathrm{B}}$ for the $\mathrm{Fe}-\mathrm{C}_{18}$ or $\mathrm{Co}-\mathrm{C}_{18}$, which is reduced compared with that of the isolated atom. This reduction results from the orbital hybridization between the TM atom and the GS. For Ni- $\mathrm{C}_{18}$, however, the total moment and the local moment located on each $\mathrm{C}$ and $\mathrm{Ni}$ atom are found to be $0 \mu_{\mathrm{B}}$. This is because the interaction of $\mathrm{Fe}$ to $\mathrm{Co}$ and $\mathrm{Ni}$ with graphene gradually increases, and the magnetic moment of $\mathrm{Ni}$ is completely quenched because of the strong interaction. The increase in binding energy from $\mathrm{Fe}$ to $\mathrm{Co}$ to $\mathrm{Ni}$ can be understood from the corresponding decrease in the interconfigurational energies, which were determined to be $0.87,0.42$, and $-0.03 \mathrm{eV},{ }^{45}$ respectively, for $\mathrm{Fe}, \mathrm{Co}$, and $\mathrm{Ni}$ when their electronic states change from $3 d^{n-2} 4 s^{2}$ to $3 d^{n-1} 4 s^{1}$ (i.e., transfer of one electron from an $s$ orbital to a $d$ orbital). Because the $3 d^{n-1} 4 s^{1}$ configuration is more active than $3 d^{n-2} 4 s^{2}$, the lower interconfigurational energy cost for the $s-d$ hybridization in Co and $\mathrm{Ni}$ results in their stronger interactions with GSs.

To see the effect of supercell size on the electronic and magnetic properties of GSs supported by the TM point contact, we also performed calculations for the $4 \times 4$ supercell. The calculated results are given in Table II, which are in good agreement with previous calculations. ${ }^{39-41}$ We find that the larger supercell slightly reduces the interaction. For instance, the binding energies become $0.859,1.190$, and $1.486 \mathrm{eV}$ in

TABLE III. The adsorption energy ( $\left.E_{\mathrm{a} 2}\right)$ of FGs on TM-supported GSs, total magnetic moment $(M)$, average bond length between the TM atom and its nearing $\mathrm{C}$ atoms $\left(d_{\mathrm{TM}-\mathrm{C}}\right)$, and adsorption distance $\left(d_{\mathrm{X}-\mathrm{C}}\right)$ of the $\mathrm{FG}$ to the $\mathrm{GS}$ for the lowest energy configurations of the $(3 \times 3)$ supercell, TM- $\mathrm{C}_{18}-\mathrm{FG}$. The calculated results for the $(4 \times 4)$ supercell, TM-C $32-\mathrm{FG}$, are also given for comparison in parentheses.

\begin{tabular}{|c|c|c|c|c|c|c|c|c|c|}
\hline \multirow[b]{2}{*}{ System } & \multicolumn{3}{|c|}{ TM-S-OH } & \multicolumn{3}{|c|}{ TM-S-CHO } & \multicolumn{3}{|c|}{ TM-S-COOH } \\
\hline & $\mathrm{Fe}$ & Co & $\mathrm{Ni}$ & $\mathrm{Fe}$ & $\mathrm{Co}$ & $\mathrm{Ni}$ & $\mathrm{Fe}$ & Co & $\mathrm{Ni}$ \\
\hline$E_{\mathrm{a} 2}$ & 1.314 & 1.758 & 1.543 & $\begin{array}{c}0.741 \\
0713)\end{array}$ & $\begin{array}{c}0.701 \\
0653)\end{array}$ & $\begin{array}{l}0.452 \\
0.409)\end{array}$ & 0.852 & 0.926 & $\begin{array}{r}0.619 \\
0507)\end{array}$ \\
\hline $\begin{array}{l}(\mathrm{eV}) \\
M\end{array}$ & $\begin{array}{c}(1.632) \\
3.00\end{array}$ & $\begin{array}{c}(1.628) \\
2.000\end{array}$ & $\begin{array}{c}(1.41 /) \\
0.505\end{array}$ & $\begin{array}{c}(0 . / 13) \\
3.00\end{array}$ & $\begin{array}{c}(0.653) \\
2.000\end{array}$ & $\begin{array}{c}(0.409) \\
0.769\end{array}$ & $\begin{array}{c}(0.834) \\
3.000\end{array}$ & $\begin{array}{c}(0.847) \\
2.000\end{array}$ & $\begin{array}{c}(0.597) \\
0.626\end{array}$ \\
\hline$\left(\mu_{\mathrm{B}}\right)$ & $(3.00)$ & $(1.82)$ & $(0.454)$ & $(3.00)$ & (1.950) & $(0.726)$ & $(2.956)$ & (1.950) & $(0.576)$ \\
\hline$d_{\mathrm{TM}-\mathrm{C}}$ & 2.227 & 2.170 & 2.181 & 2.218 & 2.176 & 2.497 & 2.463 & 2.174 & 2.187 \\
\hline$(\AA)$ & $(2.177)$ & $(2.178)$ & $(2.230)$ & $(2.181)$ & (2.173) & $(2.181)$ & $(2.201)$ & $(2.175)$ & $(2.182)$ \\
\hline$d_{\mathrm{X}-\mathrm{C}}$ & 1.477 & 1.481 & 1.481 & 1.570 & 1.569 & 1.575 & 1.573 & 1.574 & 1.578 \\
\hline$(\AA)$ & (1.480) & $(1.482)$ & (1.479) & (1.569) & (1.567) & (1.569) & $(1.571)$ & (1.571) & $(1.573)$ \\
\hline
\end{tabular}



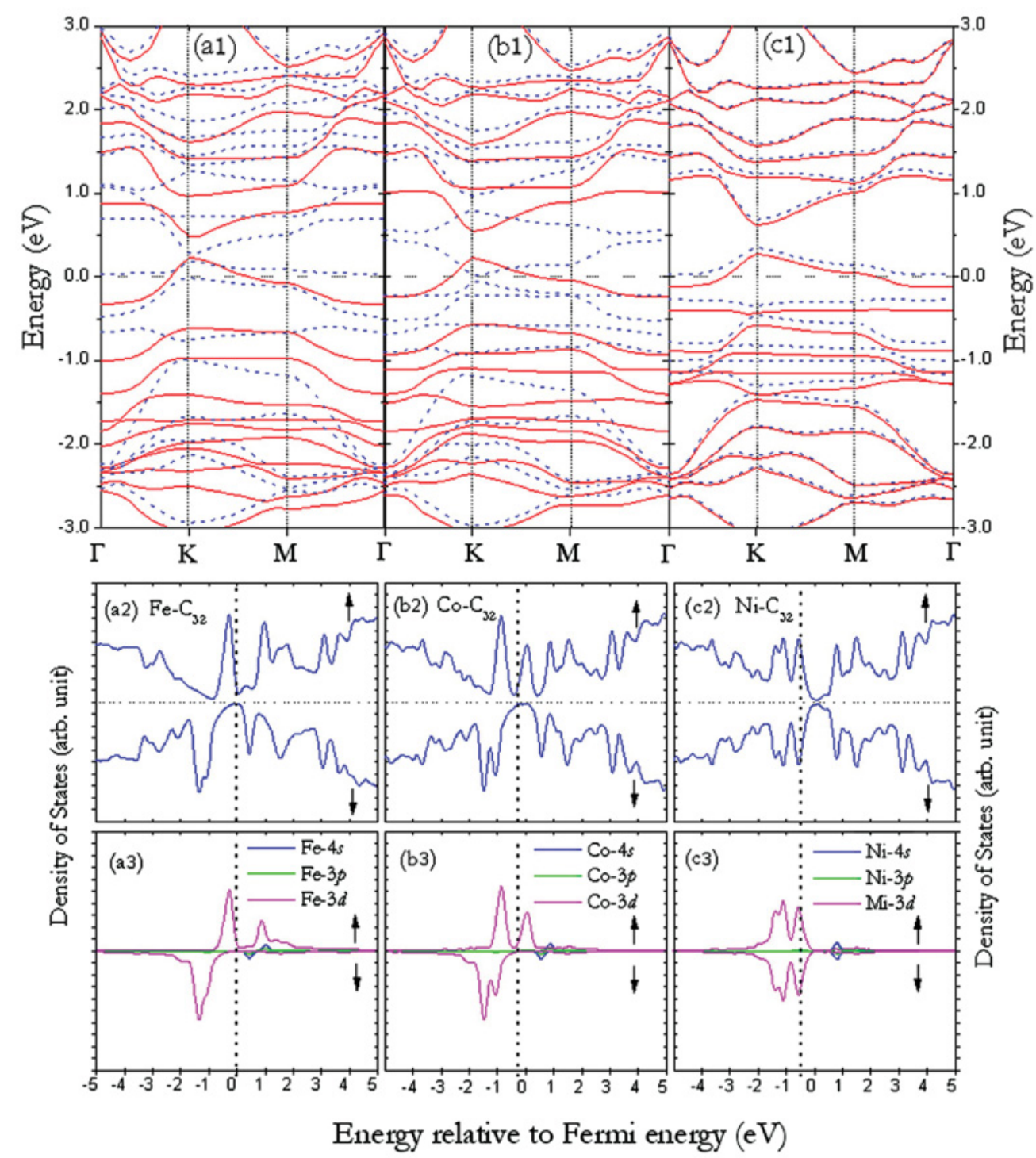

FIG. 6. (Color online) (a1), (b1), (c1) Calculated band structures for the majority (red solid lines) and minority (blue dotted lines) spin configurations, (a2), (b2), (c2) the corresponding spin-polarized total DOS, and (a3), (b3), (c3) partial DOS for the $\mathrm{Fe}-\mathrm{C}_{32}-\mathrm{CHO}, \mathrm{Co}-\mathrm{C}_{32}-\mathrm{CHO}$, and $\mathrm{Ni}-\mathrm{C}_{32}-\mathrm{CHO}$ supercells, respectively.

the $\mathrm{TM}-\mathrm{C}_{32}$ supercell for $\mathrm{TM}=\mathrm{Fe}, \mathrm{Co}$, and $\mathrm{Ni}$, respectively, which are very close to the values of $0.852,1.237$, and $1.518 \mathrm{eV}$ obtained by Liu et $a l .{ }^{41}$ using the same size supercell and same level of DFT calculation. The charge distribution on the GS supported by an Fe point contact is shown in Fig. 3(a). The results for Co- and Ni-supported GSs are quite similar. In comparing the results of OH-GS in Fig. 2(b1), we see that $\mathrm{Fe}$ capping on the $\mathrm{H}$ site has less effect on the covalent bonding features in the graphene substrate. This is because the transferred charges from $\mathrm{Fe}$ are shared by the neighboring six $\mathrm{C}$ atoms, whereas in the $-\mathrm{OH}$ case with the top-site adsorption, the charge transfer is mainly between one $\mathrm{C}$ site and the $\mathrm{O}$. The spin density distribution in Fig. 3(b) shows that the magnetic moment mainly originates from the $\mathrm{Fe}$ atom, whereas its neighboring carbon atoms are polarized antiferromagnetically, carrying small magnetic moments.

The electron ionization potential of $\mathrm{Fe}, \mathrm{Co}$, and $\mathrm{Ni}$ atoms is $7.90,7.88$, and $7.64 \mathrm{eV}$, respectively, which is larger than the work function of $4.50 \mathrm{eV}$ for GSs, so it seems that charges would transfer from graphene to the TM atoms. However, such a simple picture does not apply in this TM-GS system because of the complex chemical interactions between them. As a matter of fact, we find that the shift in Fermi energy $\Delta E_{\mathrm{F}}$ with respect to the pristine GS, defined as $\Delta E_{\mathrm{F}}=E_{\mathrm{F}}(\mathrm{TM}-\mathrm{G})-$ $E_{\mathrm{F}}(\mathrm{G})$ is $0.626,0.617$, and $0.593 \mathrm{eV}$, respectively, for $\mathrm{TM}=$ $\mathrm{Fe}, \mathrm{Co}$, and $\mathrm{Ni}$, indicating that charges are actually transferred to the GS from the TM atoms. Charge partitioning analysis reveals that about $0.2 e$ are transferred from the TM atoms to GSs. This result is in good agreement with that obtained from the double-numerical basis set linear combination of atomic orbital-based DFT calculations. ${ }^{34}$ This phenomenon has also been observed in metal surface-supported graphene. ${ }^{36}$ The band structure and spin-polarized density of states DOS are given in Fig. 4. We see that the band structures are significantly changed when the GS is supported on the Fe, Co, and Ni atoms, respectively, because of strong hybridization between the TM 
atoms and the GS. Figure 4 clearly shows that the Fe-GS is half metallic, whereas the Co-GS and Ni-GS are metallic. The Co-GS is also half metallic. The three systems have different exchange splitting, which follows the trend: Fe-GS $>$ Co-GS $>$ Ni-GS (zero for the Ni case). The partial DOS shows that, for the $\mathrm{Fe}$ - and Co-adsorbed systems, the $3 d$ orbitals dominate the majority spin states around $E_{\mathrm{F}}$, and for the Ni-adsorbed system, $\mathrm{Ni} 3 d$ orbitals dominate both the majority and minority states around the $E_{\mathrm{F}}$.

\section{Adsorption of FGs on the GS supported by TM point contact}

We then introduce the FGs to the GS supported by TM point contact $(\mathrm{TM}=\mathrm{Fe}, \mathrm{Co}$, and $\mathrm{Ni}$ ) to study their interaction. Extensive calculations were performed on the TM-GS-FG system for different adsorption sites and orientations of the FGs. We found that the six $\mathrm{C}$ atoms that are directly bonded with the TM are more reactive, providing possible adsorption sites for the FGs. Consequently, the FGs were found to prefer the carbon $\mathrm{T}$ sites rather than the $\mathrm{H}$ or $\mathrm{B}$ sites. We note that the total energy of each configuration also depends on the orientation of FGs at the $\mathrm{C}$ sites; the lowest energy configuration corresponds to when a $\mathrm{FG}$ is approximately parallel to the $\mathrm{C}-\mathrm{C}$ bond in the GS hexagon. The lowest energy configuration of $\mathrm{Fe}-\mathrm{C}_{32}-\mathrm{OH}$ is given in Fig. 5. Co- $\mathrm{C}_{32}-\mathrm{OH}$ and $\mathrm{Ni}-\mathrm{C}_{32}-\mathrm{OH}$, have similar configurations.

Although the bond distance between the $\mathrm{C}$ and the GS is reduced only by $2.0 \% \sim 3.7 \%$ compared with the unsupported cases, in all the cases, the adsorption energy is significantly enhanced to $0.741,0.701$, and $0.452 \mathrm{eV}$ for $-\mathrm{CHO}$ and to $0.852,0.926$, and $0.619 \mathrm{eV}$ for $-\mathrm{COOH}$ when they are on the Fe-, Co-, and Ni-supported GSs, respectively. We note that the adsorption energies for the unsupported graphene are small, namely, $0.033 \mathrm{eV}$ for $-\mathrm{CHO}$ and $0.025 \mathrm{eV}$ for $-\mathrm{COOH}$. The underlying reasons are the following: (1) The balance of geometric distortions from the TM on one side and the FG on the other side reduces the energy costs of geometric distortion, giving a large energy gain in the adsorption. (2) Because of charge transfer, TM support activates the $\mathrm{C}$ sites and leads to better adsorption. The main results are summarized in Table III. Compared with the conventional method of introducing defects to enhance the adsorption of -CHO or - $\mathrm{COOH}$, we can see that point contact support provides a new way to produce a patterned functionalization of GSs, and the pattern can be modulated by changing the configuration of point support underneath the GS. Furthermore, we found that when these FGs are adsorbed on the Fe-, Co-, and Ni-supported GSs, all of their corresponding Fermi levels shifted downward, suggesting that all three FGs behave like electron acceptors, which is different from the situations in unsupported GSs. The main reason is that the work function of graphene is significantly reduced when bonded with TM atoms, ${ }^{37}$ so it becomes easier to withdraw electrons. Furthermore, we find that the adsorption of FGs increases the total moment of the supercell; for $\mathrm{Fe}$ - and $\mathrm{Co}$-supported systems, the total moments are 3 and $2 \mu_{\mathrm{B}}$, respectively, whereas it is in the range of $0.5 \sim 0.8 \mu_{\mathrm{B}}$ for the Ni-supported case.

To study the effect of concentration of TM atoms on adsorption, we carried out additional calculations for the $4 \times 4$ supercell. It was found that the effect of concentration on

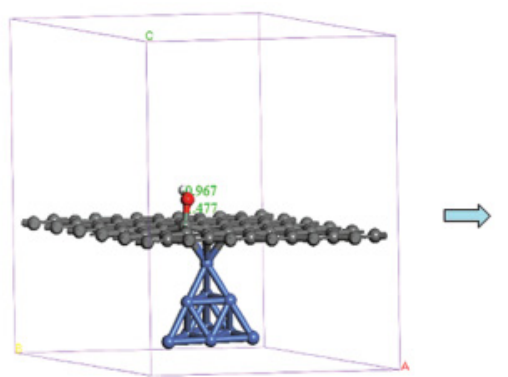

(a1)

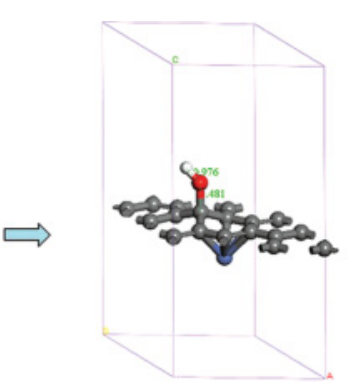

(b1)

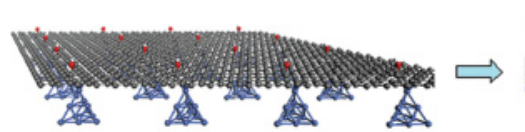

(a2)

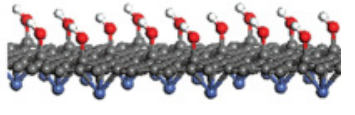

(b2)
FIG. 7. (Color online) (a1), (b1) Supercell structures of GSs supported on the Ni-pyramid and interacting with -OH, and a single $\mathrm{Ni}$ atom used as a point contact for GSs interacting with $-\mathrm{OH}$, respectively. (a2), (b2) Their corresponding extended structures. The unit of the bond lengths in (a1) and (b1) is $\AA$.

electronic and magnetic properties is not significant in this dilute limit when the concentration changes from $5.6 \%$ in a $3 \times 3$ supercell (TM-C $\left.{ }_{18}-\mathrm{FG}\right)$ to $3.1 \%$ for a $4 \times 4$ supercell $\left(\mathrm{TM}-\mathrm{C}_{32}\right.$ FG). This is because the distance between the TM atom and its mirror image in the neighboring supercells is large enough to prevent them from interacting strongly with each other. For instance, the distance between the Fe atoms in the neighboring supercells is $7.83 \AA$ for the $3 \times 3$ supercell, whereas it is 9.84 $\AA$ in the $4 \times 4$ supercell. The adsorption energy changes to $0.713,0.653$, and $0.409 \mathrm{eV}$ from $0.741,0.701$, and $0.452 \mathrm{eV}$

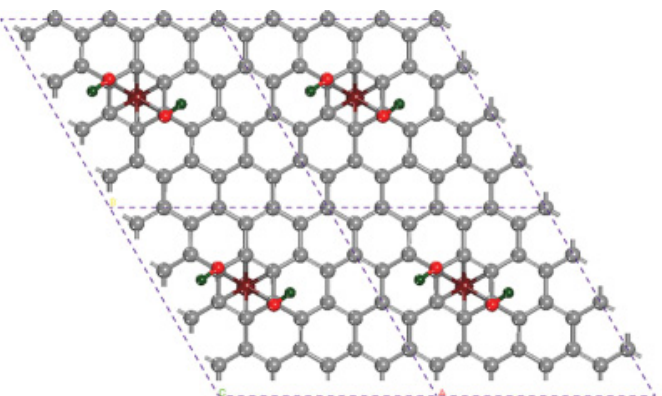

(a)

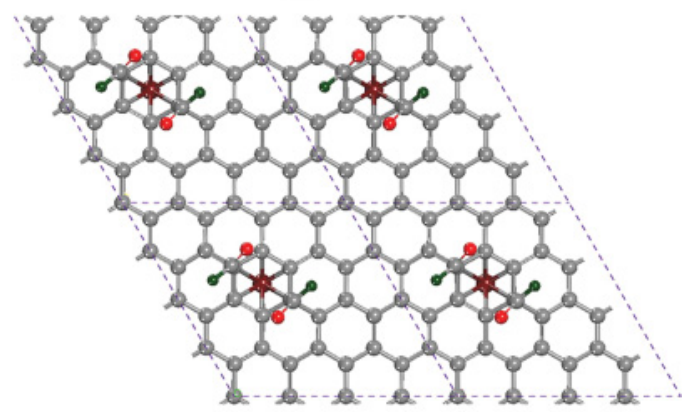

(b)

FIG. 8. (Color online) The optimized geometries of GSs supported by $\mathrm{Fe}$ point contact and interacting with two - $\mathrm{OH}$ groups (a) and with two - $\mathrm{CHO}$ groups (b). The supercells are marked by the rhombus. 
TABLE IV. The adsorption energy $E_{\mathrm{a}}$, total magnetic moment $M$, average bond length between the TM and its nearing C atoms $d_{\mathrm{TM}-\mathrm{C}}$, adsorption distance $d_{\mathrm{X}-\mathrm{C}}$, and Fermi energy $E_{\mathrm{F}}$ for the lowest energy configuration of the GS supported by TM point contact and interacting with two FGs, TM- $\mathrm{C}_{32}-2 \mathrm{FG}(\mathrm{TM}=\mathrm{Fe}, \mathrm{Co}$, and $\mathrm{Ni})$.

\begin{tabular}{|c|c|c|c|c|c|c|c|c|c|}
\hline & \multicolumn{3}{|c|}{$\mathrm{TM}-\mathrm{C}_{32}-2 \mathrm{OH}$} & \multicolumn{3}{|c|}{$\mathrm{TM}^{-\mathrm{C}_{32}}-2 \mathrm{CHO}$} & \multicolumn{3}{|c|}{ TM-C $32-2 \mathrm{COOH}$} \\
\hline & $\mathrm{Fe}$ & $\mathrm{Co}$ & $\mathrm{Ni}$ & $\mathrm{Fe}$ & $\mathrm{Co}$ & $\mathrm{Ni}$ & $\mathrm{Fe}$ & $\mathrm{Co}$ & $\mathrm{Ni}$ \\
\hline$E_{\mathrm{a}}(\mathrm{eV} / \mathrm{FG})$ & 1.470 & 1.535 & 1.517 & 0.633 & 0.729 & 0.631 & 0.789 & 0.870 & 0.724 \\
\hline$M\left(\mu_{\mathrm{B}}\right)$ & 2.092 & 1.000 & 0.000 & 2.062 & 1.000 & 0.000 & 2.053 & 1.000 & 0.000 \\
\hline$d_{\mathrm{TM}-\mathrm{C}}(\AA)$ & 2.243 & 2.202 & 2.222 & 2.239 & 2.202 & 2.205 & 2.239 & 2.239 & 2.217 \\
\hline$d_{\mathrm{X}-\mathrm{C}}(\AA)$ & 1.471 & 1.473 & 1.470 & 1.573 & 1.572 & 1.575 & 1.567 & 1.572 & 1.572 \\
\hline$E_{\mathrm{F}}(\mathrm{eV})$ & -1.894 & -2.016 & -2.096 & -1.830 & -2.020 & -2.072 & -1.533 & -1.742 & -1.672 \\
\hline
\end{tabular}

for - $\mathrm{CHO}$ on the $\mathrm{Fe}-$, $\mathrm{Co}-$, and Ni-GS, respectively, when supercell size is increased. A similar trend was found for the -COOH-containing systems; namely, the adsorption energy slightly reduces when the supercell size increases. The total magnetic moment for the larger systems was also found to be slightly smaller than that for the $(3 \times 3)$ systems. The calculated results are given in Table III. To have a better insight into the nature of bonding and electronic properties of systems in which the FGs are introduced to the TM-supported GSs, we studied the band structure and DOS for all the systems. The results are shown in Fig. 6 for the $\mathrm{TM}_{32}-\mathrm{CHO}(\mathrm{TM}=\mathrm{Fe}, \mathrm{Co}$, and $\mathrm{Ni})$ as examples. Compared with the band structures and DOS in Fig. 4, we see that the main effect of - $\mathrm{CHO}$ adsorption is that all three systems become half-metallic. We also note that there are some DOS reductions at their Fermi levels caused by the electron withdrawing charge transfer.

To further examine the adequacy of using one TM atom to mimic a point contact situation, we generated a $\mathrm{Ni}$ pyramid containing $10 \mathrm{Ni}$ atoms from a Ni (111) crystal surface, which is widely used as substrate in epitaxial experiments. We chose the $(6 \times 6)$ supercell and placed the pyramid under the graphene surface for support, as shown in Fig. 7(a1). In the calculations, we kept the six Ni atoms at the bottom of the pyramid in their bulk position and allowed all other atoms to relax without any symmetry constraints. We found that when an $-\mathrm{OH}$ is introduced, a vacuum space of $23 \AA$ in the normal direction of the supercell and the MP k-point mesh of $(11 \times 11 \times 1)$ are necessary to ensure the convergence of the system. The increase of adsorption energy of -OH when the GS was supported by the pyramid, as shown in Fig. 7(a1), is $0.76 \mathrm{eV}$, whereas that when a single $\mathrm{Ni}$ atom is used for contact, as shown in Fig. 7(b1), is $0.83 \mathrm{eV}$. The differences of both the $\mathrm{C}-\mathrm{O}$ and $\mathrm{O}-\mathrm{H}$ bond lengths in these two cases are less than $0.01 \AA$. Because the main results-namely, the adsorption energy is significantly enhanced when the GS is supported by point contact-remained unaffected, to reduce computational cost, we simplify the model of point contact shown in Figs. 7(a2) to 7(b2).

Additionally, we have studied how the properties of the TM-GS-FG system change when more FGs are introduced. For these calculations, we used the $4 \times 4$ unit cell. We found that the favorable adsorption site is still the on-top $\mathrm{C}$ site, wherein the $\mathrm{C}$ atoms are directly bonded with the TM atoms underneath the sheet. But because of the steric hindrance between FGs, the second FG favors an orientation opposite the first one. The optimized geometries of the $-2 \mathrm{OH}$ and $-2 \mathrm{CHO}$ adsorbed on the Fe-supported GS, Fe- $\mathrm{C}_{32}-2 \mathrm{OH}$ and
Fe- $\mathrm{C}_{32}-2 \mathrm{CHO}$, are shown in Figs. 8(a) and 8(b), respectively. It is found that the adsorption distance and the average bond length between the TM atom and its nearest six $\mathrm{C}$ atoms almost remain the same as those when only one $\mathrm{FG}$ is adsorbed, whereas the graphene distortion is slightly smaller. The change in adsorption energy, on the other hand, is system dependent. The adsorption energy increases to 0.729 and $0.631 \mathrm{eV} / \mathrm{FG}$ for $\mathrm{Co}-\mathrm{C}_{32}-2 \mathrm{CHO}$ and $\mathrm{Ni}-\mathrm{C}_{32}-2 \mathrm{CHO}$, respectively. We recall that the corresponding energies when only one FG is adsorbed are 0.653 and $0.409 \mathrm{eV} / \mathrm{FG}$, respectively. For $\mathrm{Co}-\mathrm{C}_{32}-2 \mathrm{COOH}$ and $\mathrm{Ni}-\mathrm{C}_{32}-2 \mathrm{COOH}$, the adsorption energy changes to 0.870 and $0.724 \mathrm{eV} / \mathrm{FG}$ from 0.834 and $0.597 \mathrm{eV} / \mathrm{FG}$, respectively. It slightly decreases for the

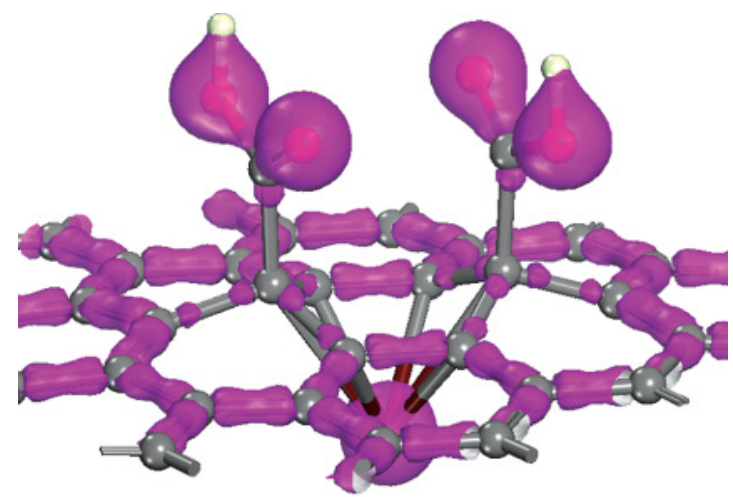

(a)

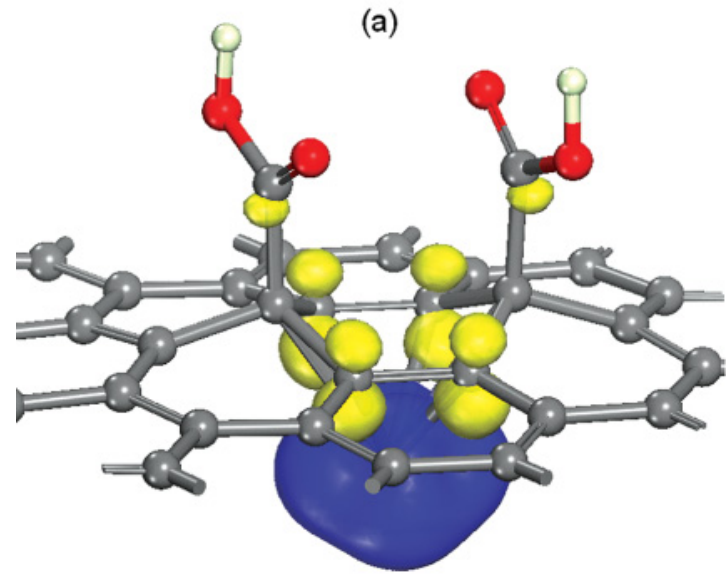

(b)

FIG. 9. (Color online) (a) The charge density isosurface with a value of 1.85 electrons $/ \AA^{3}$ and (b) the corresponding 3D spin density isosurface with a value of 0.03 electrons $/ \AA^{3}$ for the $\mathrm{Fe}-\mathrm{C}_{32}-2 \mathrm{COOH}$. 


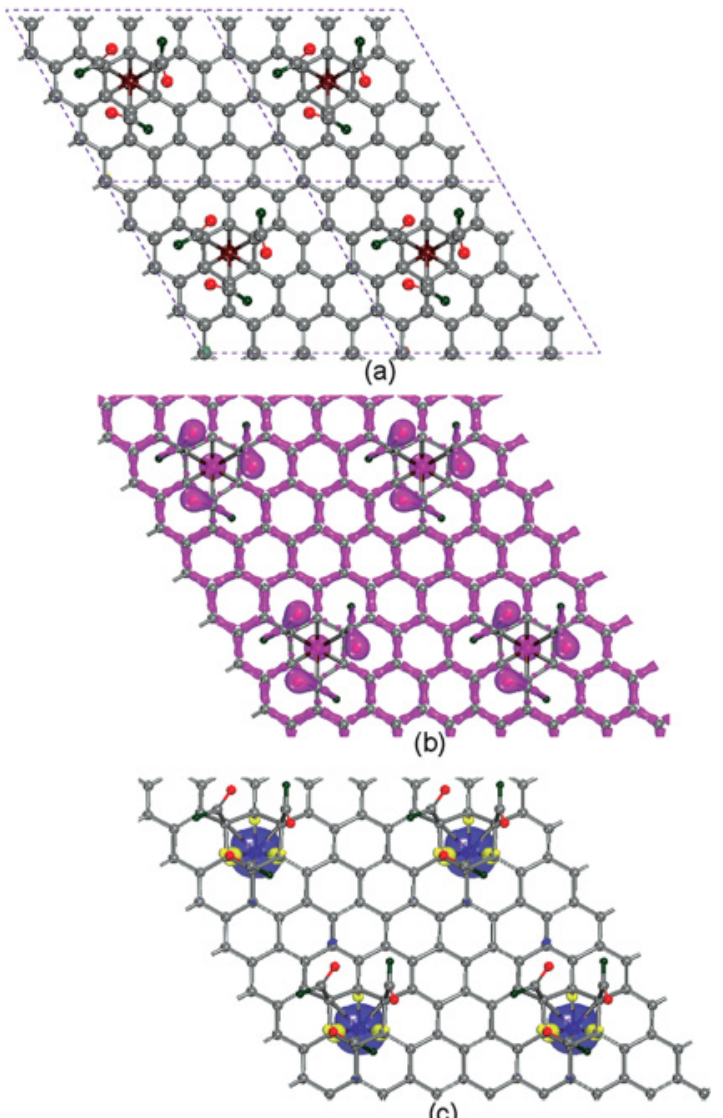

(c)

FIG. 10. (Color online) (a) The optimized geometry, (b) charge density isosurface with a value of 1.85 electrons $/ \AA^{3}$, and (c) corresponding spin density isosurface with a value of 0.04 electrons $/ \AA^{3}$ for $\mathrm{Fe}-\mathrm{C}_{32}-3 \mathrm{CHO}$. The supercell is marked by the rhombus.

Fe-supported systems by $0.16,0.11$, and $0.06 \mathrm{eV} / \mathrm{FG}$ for $-2 \mathrm{OH}$, $-2 \mathrm{CHO}$, and $-2 \mathrm{COOH}$, compared with the binding with $-\mathrm{OH}$, $-\mathrm{CHO}$, and $-\mathrm{COOH}$, respectively. Moreover, the magnetic moment is also changed because of increased coverage. The calculated total moments are about 2,1 , and $0 \mu_{\mathrm{B}}$ for the Fe-, Co-, and Ni-supported systems, respectively. Table IV gives the detailed data. The charge density isosurface and its corresponding 3D spin density isosurface for $\mathrm{Fe}-\mathrm{C}_{32}-2 \mathrm{COOH}$ are plotted in Figs. 9 as an example. We can see that when the graphene sheet is supported on $\mathrm{Fe}$ atoms, the six $p_{z}$ orbitals of $\mathrm{C}$ atoms are antiferromagnetically polarized [see Fig. 3(b)], when two - $\mathrm{COOH}$ are introduced; two of the $\mathrm{C}$ sites in the graphene sheet become $s p^{3}$ bonded; the left four $p_{\mathrm{z}}$ orbitals are still antiferromagnetically polarized; and meanwhile, the two $\mathrm{C}$ in two - $\mathrm{COOH}$ are also slightly polarized [see Fig. 9(b)].

We have also studied the adsorption of three - CHO by adding one more to $\mathrm{TM}-\mathrm{C}_{32}-2 \mathrm{CHO}$ ( $\mathrm{TM}=\mathrm{Fe}, \mathrm{Co}$, and $\mathrm{Ni})$. The optimized geometry, charge density isosurface, and corresponding spin density isosurface are plotted in Figs. 10(a), 10(b), and 10(c), respectively. It was found that in the most stable configuration, these three - $\mathrm{CHO}$ are patterned and uniformly distributed on the hexagonal ring directly bonding with the TM. The geometric parameters for Fe- and Co-supported systems have no obvious changes. For the $\mathrm{Ni}$-supported system, the average bond length between the $\mathrm{Ni}$ and its nearing $\mathrm{C}$ atoms increases from 2.205 to $2.236 \AA$. Consequently, the adsorption energy changes from 0.631 to $0.525 \mathrm{eV}$ (i.e., the strength of adsorption of the FG on the Ni-supported GS is slightly reduced as more FGs are introduced). The Fermi energies are downward shifted from $-1.830,-2.020$, and $-2.072 \mathrm{eV}$ to $-2.081,-2.321$, and $-2.111 \mathrm{eV}$, respectively, when going from $\mathrm{TM}-\mathrm{C}_{32}-2 \mathrm{CHO}$ to $\mathrm{TM}-\mathrm{C}_{32}-3 \mathrm{CHO}$, suggesting again that more electrons are withdrawn by the FGs. From the discussion above, we have seen that the support can significantly affect functionalization, similar to that found in graphene deposited on a SiC surface. ${ }^{28}$

\section{CONCLUSIONS}

Using DFT calculations, we have performed systematic studies of the adsorptions of $-\mathrm{OH},-\mathrm{CHO}$, and $-\mathrm{COOH}$ on pristine graphene and supported graphene. Following are the main conclusions of our work: (1) -OH can strongly bind with the free-standing GS, whereas the energy gains for binding $-\mathrm{CHO}$ or $-\mathrm{COOH}$ are small because of the energy cost caused by geometric distortions. (2) When a GS is supported by Fe, $\mathrm{Co}$, and $\mathrm{Ni}$ atoms as point contacts, the hollow site is the most preferable site, and charges are transferred to the GS from metal atoms. The magnetic moment of $\mathrm{Ni}$ is totally quenched, and the moments of $\mathrm{Fe}$ and $\mathrm{Co}$ are reduced to 2 and $1 \mu_{\mathrm{B}}$, respectively. (3) When the GS is supported on metal atoms, the carbon atoms bonded with the TM atom are activated, resulting in enhanced adsorptions of - $\mathrm{CHO}$ and $-\mathrm{COOH}$, as well as - $\mathrm{OH}$. The activated $\mathrm{C}$ sites on the sheet provide the preferred sites for adsorption and thus can be used for the synthesis of patterned structures. The pattern can be further tuned by changing the shape, size, and distribution of the supports underneath the GS. Furthermore, because of the reduced work function in GSs supported by TM point contact, the structure is more flexible, and the electrons are more easily manipulated for fine tuning the electronic structure and properties. (4) On the free-standing sheet, $-\mathrm{OH}$ acts as an electron acceptor, withdrawing electrons from the sheet, whereas $-\mathrm{CHO}$ and $-\mathrm{COOH}$ behave like electron donors, giving electrons to the sheet. However, on the supported GS, all three of these radicals become electron acceptors. This is very different from the situation with benzene molecules, wherein - $\mathrm{OH}$ donates electrons and $-\mathrm{CHO}$ and $-\mathrm{COOH}$ withdraw electrons. (5) Because of charge redistribution, covalent bonding in the GS is weakened once the metal atom or the radical is introduced. The present study sheds new light on better understanding the interactions of radicals with GSs in various environments.

\section{ACKNOWLEDGMENTS}

This work is partially supported by grants from the National Natural Science Foundation of China (Grant No. NSFC11174014), the National Grand Fundamental Research 973 Program of China (Grant No. 2012CB921404), and the US Department of Energy. The authors thank the crew of the Center for Computational Materials Science, the Institute for Materials Research, Tohoku University (Japan), for their continuous support of the HITACH SR 11000 supercomputing facility. One of the authors, Y. Kawazoe, is thankful to the CREST project, headed by Motoko Kotani, for support from mathematicians. 
"qianwang2@pku.edu.cn

${ }^{1}$ A. H. Castro Neto, F. Guinea, N. M. R. Peres, K. S. Novoselov, and

A. K. Geim, Rev. Mod. Phys. 81, 109 (2009).

${ }^{2}$ M. J. Allen, V. C. Tung, and R. B. Kaner, Chem. Rev. 110, 132 (2010)

${ }^{3}$ A. K. Geim, Science 324, 1530 (2009).

${ }^{4}$ D. C. Elias, R. R. Nair, T. M. G. Mohiuddin, S. V. Morozov, P. Blake, M. P. Halsall, A. C. Ferrari, D. W. Boukhvalov, M. I. Katsnelson, A. K. Geim, and K. S. Novoselov, Science 323, 610 (2009).

${ }^{5}$ J. Zhou, Q. Wang, Q. Sun, X. S. Chen, Y. Kawazoe, and P. Jena, Nano Lett. 9, 3867 (2009).

${ }^{6}$ G. Wang, X. Shen, B. Wang, J. Yao, and J. Park, Carbon 47, 1359 (2009).

${ }^{7}$ E. Varrla, B. Krishnan, and R. Sundara, J. Mat. Chem. 34, 12626 (2011).

${ }^{8}$ M. Castelaín, H. J. Salavagione, R. Gómez, and J. L. Segura, Chem. Comm. 47, 7677 (2011).

${ }^{9}$ S. Stankovich, D. A. Dikin, G. H. B. Dommett, K. M. Kohlhaas, E. J. Zimney, E. A. Stach, R. D. Piner, S. T. Nguyen, and R. S. Ruoff, Nature 442, 282 (2006).

${ }^{10}$ D. W. Boukhvalov and M. I. Katsnelson, Nano Lett. 8, 4373 (2008).

${ }^{11}$ A. Bagri, R. Grantab, N. V. Medhekar, and V. B. Shenoy, J. Phys. Chem. C 114, 12053 (2010).

${ }^{12}$ F. OuYang, B. Huang, Z. Li, J. Xiao, H. Wang, and H. Xu, J. Phys. Chem. C 112, 12003 (2008).

${ }^{13}$ A. A. Nabil and V. Igor, J. Phys. Chem. C 113, 12970 (2009).

${ }^{14}$ S. Dutta and S. K. Pati, J. Mater. Chem. 20, 8207 (2010).

${ }^{15} \mathrm{~N}$. Gorjizadeh and Y. Kawazoe, J. Nanomater. 10, 513501 (2010).

${ }^{16}$ D. W. Boukhvalov and M. I. Katsnelson, Phys. Rev. B 78, 085413 (2008).

${ }^{17}$ G. Kresse and D. Joubert, Phys. Rev. B 59, 1758 (1999).

${ }^{18}$ G. Kresse and J. Furthmüller, Phys. Rev. B 54, 11169 (1996).

${ }^{19}$ Y. Wang and J. P. Perdew, Phys. Rev. B 44, 13298 (1991).

${ }^{20}$ H. J. Monkhorst and J. D. Pack, Phys. Rev. B 13, 5188 (1976).

${ }^{21}$ R. J. W. E. Lahaye, H. K. Jeong, C. Y. Park, and Y. H. Lee, Phys. Rev. B 79, 125435 (2009).

${ }^{22}$ M. C. Kim, G. S. Hwang, and R. S. Ruoff, J. Chem. Phys. 131, 064704 (2009).
${ }^{23}$ W. F. Li, M. W. Zhao, Y. Y. Xia, R. Q Zhang, and Y. G. Mu, J. Mater. Chem. 19, 9274 (2009).

${ }^{24}$ N. Ghderi and M. Peressi, J. Phys. Chem. C 114, 21625 (2010).

${ }^{25}$ D. W. Boukhvalov and M. I. Katsnelson, J. Phys. D: Appl. Phys. 43, 175302 (2010).

${ }^{26}$ D. W. Boukhvalov, Phys. Chem. Chem. Phys. 12, 15367 (2010).

${ }^{27}$ D. W. Boukhvalov and M. I. Katsnelson, ACS Nano 5, 2440 (2011).

${ }^{28}$ D. W. Boukhvalov, Nanotechnology 22055708 (2011).

${ }^{29}$ F. Toda, Eur. J. Org. Chem. 8, 1377 (2000).

${ }^{30}$ J. J. Novoa, P. Lafuente, R. E. Del Sesto, and J. S. Miller, Angew. Chem. Int. Ed 40, 2540 (2001).

${ }^{31}$ S. Suzuki, Y. Morita, K. Fukui, K. Sato, D. Shiomi, T. Takui, and K. Nakasuji, J. Am. Chem. Soc. 128, 2530 (2006).

${ }^{32}$ O. V. Mykhailenko, Yu. I. Prylutskyy, D. Matsui, Y. M. Strzhemechny, F. Le Normand, U. Ritter, and P. Scharff, J. Comput and Theor. Nanosci. 7, 996 (2010).

${ }^{33}$ J. M. Lee, Nano Lett. 10, 2783 (2010).

${ }^{34}$ H. Johll, H. C. Kang, and E. S. Tok, Phys. Rev. B 79, 245416 (2009),

${ }^{35}$ Y. Yagi, T. M. Briere, M. H. F. Sluiter, V. Kumar, A. A. Farajian, and Y. Kawazoe, Phys. Rev. B 69, 075414 (2004).

${ }^{36}$ H. Valencia, A. Gil, and G. Frapper, J. Phys. Chem. C 11414145 (2010).

${ }^{37}$ C. Cao, M. Wu, J. Z. Jiang, and H.-P. Cheng, Phys. Rev. B 81, 205424 (2010).

${ }^{38}$ H. Sevinçli, M. Topsakal, E. Durgun, and S. Ciraci, Phys. Rev. B 77, 195434 (2008)

${ }^{39}$ K. T. Chan, J. B. Neaton, and M. L. Cohen, Phys. Rev. B 77, 235430 (2008).

${ }^{40}$ Y. L. Mao, J. M. Yuan, and J. X. Zhong, J. Phys.: Condens. Matter 20, 115209 (2008).

${ }^{41}$ X. J. Liu, C. Z. Wang, Y. X. Yao, W. C. Lu, M. Hupalo, M. C. Tringides, and K. M. Ho, Phys. Rev. B 83, 235411 (2011).

${ }^{42}$ K. T. Chan, H. K. Lee, and M. L. Cohen, Phys. Rev. B 83, 035405 (2011).

${ }^{43}$ L. Hu, X. Hu, X. Wu, C. Du, Y. Dai, and J. Deng, Physica B 405, 3337 (2010).

${ }^{44}$ D. M. Duffy and J. A. Blackman, Phys. Rev. B 58, 7443 (1998).

${ }^{45}$ G. Giovannetti, P. A. Khomyakov, G. Brocks, V. M. Karpan, J. van den Brink, and P. J. Kelly, Phys. Rev. Lett. 101, 026803 (2008). 